\title{
Heat transfer convection of a power law fluid flow within a parallel plate channel provided with two generating obstacles
}

\author{
Ahlem Boudiaf*, Fetta Danane, Youb Khaled Benkahla, Nabila Labsi, and Abdelkader Boutra \\ Faculty of Mechanical and Process Engineering, University of Science and Technology Houari Boumediene, \\ USTHB, Algiers, Algeria
}

Received: 17 July 2016 / Accepted: 15 September 2017

\begin{abstract}
The main objective of this work is to examine the heat transfer changes induced by the presence of heat generating obstacles and that for non-Newtonian fluid flow within a parallel plate channel. The effects of the two obstacle height and the distance between them, on the flow structure and Nusselt number are examined. The Finite Volume Method is used to discretize the conservation equations of mass, momentum, and energy. The SIMPLER algorithm is applied to remove the checkerboard pressure problem. The results are discussed in terms of streamlines and the Nusselt number for three combinations of height and separation distance of the two obstacles. Two recirculation zones are observed for all the cases with different intensity varying with the size of the obstacle and the separation distance. Likewise the results show that the heat removal, occurring by the Nusselt number variations, is widely affected by the size of the obstacles as well as by the distance between them.
\end{abstract}

Keywords: Heat generating obstacles / non-Newtonian fluid / finite volume method

\section{Introduction}

During the last few years, numerous studies have shown that heat transfer enhancement can be achieved by creating longitudinal vortices in the flow. These vortices are created by introducing an obstacle within the flow, which can provide additional heat transfer surface area. Among these works, we quote Young and Vafai [1,2] who investigated the forced convection heat transfer of individual and array of multiple two-dimensional obstacles. The effect of a change in the obstacle height, width as well as the thermal conductivity was investigated and an empirical correlation was established. Through the use of a stream function vorticity transformation, Yang et al. [3] studied the influence of the aspect ratios of fins, the Reynolds number and the thermal conductivity ratio. They found that the optimum aspect ratio of a fin, corresponding to the fin with a maximum heat transfer rate, increases with increasing the Reynolds number but decreases with the thermal conductivity ratio.

Hamouche and Bessaih [4] studied laminar mixed convection in channel provided with two identical blocks. They found that the overall heat transfer increases as the height and the width of the obstacle increase. In addition, correlations were proposed to calculate the average Nusselt number of the first and the second obstacle.

\footnotetext{
* Corresponding author: e-mail: ahlem.boudiaf@gmail.com
}

Kim et al. [5] investigated pulsating flow and heat transfer characteristics from two heated blocks in a channel. The effects of Reynolds number, Strouhal number, pulsation amplitude and the spacing between two blocks are investigated.

Korichi and Oufer [6] studied numerically the heat transfer convection between a fluid and three obstacles. The dependence of the flow and the temperature fields on parametric changes such as the Reynolds number, the solid thermal conductivity and the geometric parameters was investigated.

Using the lattice Boltzmann method, Pirouz et al. [7] carried out a numerical investigation of heat transfer in a rectangular channel with lower and upper wall-mounted obstacles. The results show that reducing the distance between the obstacles deviates and accelerates the flow near the obstacles faces and causes an increase in the rate of heat transfer from obstacles.

Another tool which has become rather popular in recent times for the enhancement of heat transfer is the use of solid element to modify flow pattern. Wu and Perng [8] used an oblique plate to generate vortex shedding in a horizontal block-heated channel. They showed that installing an oblique plate can effectively enhance the heat transfer performance.

Other applications of vortex generators to enhance heat transfer can be found in the studies of Oztop et al. [9] and Perng at $\mathrm{Wu}[10]$. 
(a): Obstacle $1=$ obstacle 2

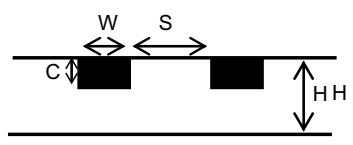

(b): Obstacle $1<$ obstacle 2

(c): Obstacle $1>$ obstacle 2

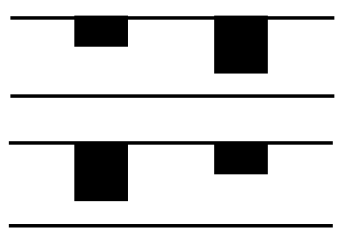

Fig. 1. Schematic of the physical domain.

However, all the cited works used Newtonian fluids while in numerous engineering applications, the fluids are non-Newtonian for which several models have been proposed. Amongst these models, the most common is that of Ostwald-De Waele, which has received special attraction from the researchers in the field. Cotta and Raleigh [11] have studied the flow of a non-Newtonian fluid obeying the Ostwald-De Waele model in a cylindrical pipe and between two parallel planes maintained at a uniform wall temperature. Their results for the Nusselt number and the average temperature of the fluid for both geometries were tabulated.

In the numerical study undertaken by Nebbali and Bouhadef [12] regarding the case of a non-Newtonian fluid moving between two parallel planes, having a porous block attached to the two planes, the authors found that the pseudoplastic fluids generate a greater heat transfer compared with the dilatants and Newtonian case. Furthermore, the presence of porous blocks causes a significant increase in pressure drop, which is found to be more important with dilatant fluids. The influence of Darcy and Reynolds numbers, the power-law index and the ratio of thermal conductivities on flow and heat transfer characteristics were also discussed.

Because of a significant lack of research on the flows of non-Newtonian fluids in presence of obstacles, this work seems more relevant than previous ones. The combined effects induced by the presence of the non-Newtonian fluid and the heat generating obstacles are analysed numerically in the present study. The influences of the obstacle height and the separation distance between the two obstacles, upon the flow and heat transfer characteristics are the main objectives of this study.

\section{Mathematical model}

The forced convection of a power-law fluid flow within a parallel plates channel with two heat generating obstacles located at the upper wall is considered in the present study. The study focuses first, on the effect of the obstacles height on the heat transfer and fluid flow. For this purpose, three combinations are taken into account, as shown in Figure 1.

Then, three values of the separation distance between the two obstacles are considered: $S=W, S=2 W$ and $S=3 W$.

The non-dimensional equations for mass, momentum and energy conservation are given in the Cartesian coordinate system as follows:

$$
\begin{aligned}
& \frac{\partial U}{\partial X}+\frac{\partial V}{\partial Y}=0 \\
& U \frac{\partial U}{\partial X}+V \frac{\partial V}{\partial Y}=-\frac{\partial P^{*}}{\partial X}+\frac{1}{R e}\left[\frac{\partial}{\partial X}\left(\phi^{(n-1) / 2} \frac{\partial U}{\partial X}\right)\right. \\
& \left.+\frac{\partial}{\partial Y}\left(\phi^{(n-1) / 2} \frac{\partial U}{\partial Y}\right)\right]+\frac{1}{R e}\left[\phi^{(n-1) / 2} \frac{\partial^{2} U}{\partial X^{2}}+\phi^{(n-1) / 2}\right. \\
& \left.\frac{\partial^{2} V}{\partial X \partial Y}+\frac{\partial \phi^{(n-1) / 2}}{\partial X} \frac{\partial U}{\partial X}+\frac{\partial \phi^{(n-1) / 2}}{\partial Y} \frac{\partial V}{\partial X}\right] \\
& U \frac{\partial V}{\partial X}+V \frac{\partial V}{\partial Y}=-\frac{\partial P^{*}}{\partial Y}+\frac{1}{R e}\left[\frac{\partial}{\partial X}\left(\phi^{(n-1) / 2} \frac{\partial V}{\partial X}\right)\right. \\
& \left.+\frac{\partial}{\partial Y}\left(\phi^{(n-1) / 2} \frac{\partial V}{\partial Y}\right)\right]+\frac{1}{R e}\left[\phi^{(n-1) / 2} \frac{\partial^{2} V}{\partial Y^{2}}+\phi^{(n-1) / 2}\right. \\
& \left.\frac{\partial^{2} U}{\partial X \partial Y}+\frac{\partial \phi^{(n-1) / 2}}{\partial Y} \frac{\partial U}{\partial X}+\frac{\partial \phi^{(n-1) / 2}}{\partial Y} \frac{\partial V}{\partial X}\right] \\
& U \frac{\partial \theta}{\partial X}+V \frac{\partial \theta}{\partial Y}=\frac{K}{\operatorname{Pr} R e}\left(\frac{\partial^{2} \theta}{\partial X^{2}}+\frac{\partial^{2} \theta}{\partial Y^{2}}\right)+\frac{\lambda}{\operatorname{Pr} \operatorname{Re} C W}
\end{aligned}
$$

All of the above variables have been dimensionless based on the following definitions:

$$
\begin{gathered}
X=\frac{x}{H}, \quad Y=\frac{y}{H}, \quad U=\frac{u}{U_{0}}, \\
V=\frac{v}{U_{0}}, \quad P^{*}=\frac{p}{\rho U_{0}^{2}}, \theta=\frac{T-T_{e}}{Q H / k_{f}}, \quad K=\frac{k_{s}}{k_{f}}
\end{gathered}
$$

where $K$ is the thermal conductivity ratio defined as:

$$
K= \begin{cases}k_{s} / k_{f} & \text { in the solid blocks } \\ 1 & \text { in the fluid domain }\end{cases}
$$

$\lambda$ is set equal to one to account for heat generation in the blocks, and to zero elsewhere.

$$
\phi^{(n-1) / 2}=\left\{2\left[\left(\frac{\partial U}{\partial X}\right)^{2}+\left(\frac{\partial V}{\partial Y}\right)^{2}\right]+\left[\frac{\partial U}{\partial Y}+\frac{\partial V}{\partial X}\right]^{2}\right\}^{(n-1) / 2}
$$

\subsection{Boundary conditions}

The fluid is assumed to enter into the channel at the ambient temperature with fully developed parabolic profile.

$X=0$ and $0<Y<1, \quad U=6 Y(1-Y), V=0, \theta=0$ 


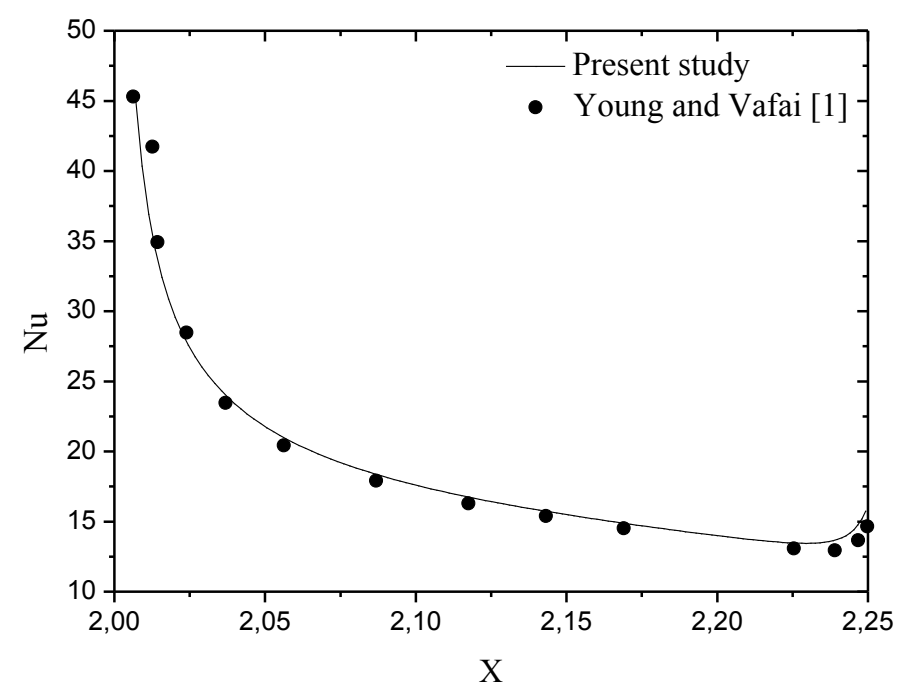

Fig. 2. Nusselt number on the obstacle top face. $R e=1000$, $\operatorname{Pr}=0.7, n=1$.

At the exit, fully developed hydrodynamic and thermal conditions are applicable.

$$
\begin{aligned}
& X=L \text { and } 0<Y<1 \\
& \frac{\partial U}{\partial X}=0, \frac{\partial V}{\partial X}=0, \frac{\partial \theta}{\partial X}=0
\end{aligned}
$$

No slip conditions are applied at both the upper and lower channel walls that are taken adiabatic.

$$
\begin{aligned}
& Y=0 \text { and } Y=1,0<X<L \\
& U=0, V=0, \frac{\partial \theta}{\partial X}=0
\end{aligned}
$$

\section{Numerical model}

The governing equations (1-4), with the associated boundary conditions, are solved using the finite volume method, as described by Patankar [13]. The algorithm SIMPLER is used to handle the pressure-velocity coupling. The discretized algebraic equations are solved by the lineby-line tri diagonal matrix algorithm (TDMA).

The study of the grid independence has been done. Nonuniform grid sizes with a dense packed near the top and bottom surfaces of the parallel plates as well as in the neighborhood of fluid/block boundaries in both directions were adopted. The $260 \times 80$ grid was used for the present physical system. The convergence is declared when the maximum relative change between two consecutive iteration level falls below $10^{-4}$, for both the velocity components ( $U$ and $V$ ) and the temperature $(\theta)$.

To validate the computer code, a comparison with the published results of Young and Vafai [1] was made; the results are in excellent agreement with a maximum discrepancy of about $3 \%$ (Fig. 2).



Fig. 3. Effect of the mesh size on the local Nusselt number for $R e=100$.

\section{Results and discussion}

The focus of this work is to investigate the effects of change in obstacles height and the distance between them, upon flow and heat transfer characteristics. All the computations were conducted for three values of the flow index: $n=0.5$, $n=1$ and $n=1.5$ and for $\operatorname{Pr}=10, R e=100$ and a thermal conductivity ratio of the obstacles $k_{s} / k_{f}=10$ (Fig. 3 ).

\section{Effect of the obstacle height on the stream function}

Figure 4 shows the streamlines for various heights of the obstacle and for a power law fluid of a flow index equal to 0.5 . We can see two recirculation zones between the two obstacles and behind the second one for both combinations (a) and (b). The height of the obstacle affects strongly the flow structure. Indeed, the streamlines of the main flow are densely packed in the region near the obstacle, particularly under the higher one.

It is interesting to note also that for the combination (b), the size and the strength of the recirculation zone behind the second obstacle is enlarged when the obstacle in question is elongated. However, for the combination (c), we can observe the formation of a single recirculation zone with a greater intensity and that extends from the region between the two obstacles to far downstream the second obstacle.

The effect of the obstacles height on flow pattern for a flow index equal $n=1$ is shown in Figure 5 . It can be seen that two recirculation zones are created close to the obstacles wall, which sizes are approximately equal to the extent of the flow blockage by the obstacles.

As for the shear-thinning $(n=0.5)$ and the Newtonian $(n=1)$ fluid, the streamlines are straight upstream and downstream the two obstacles and they are affected by the presence of the obstacle where, as we can see in Figure 6, two recirculation zones are created $(n=1.5)$. The extent 

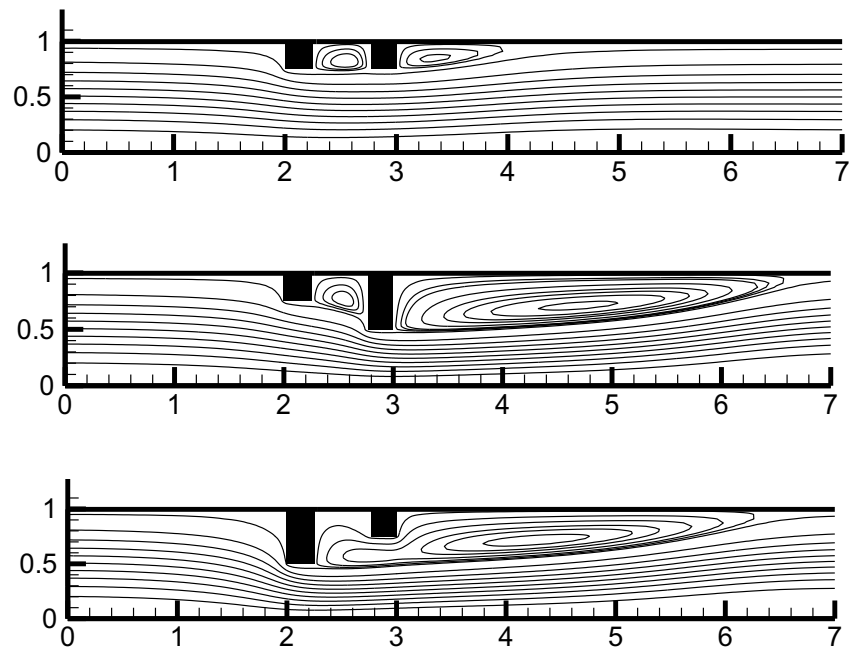

Fig. 4. Streamlines for the three combinations. $R e=100$, $\operatorname{Pr}=10, n=0.5$.
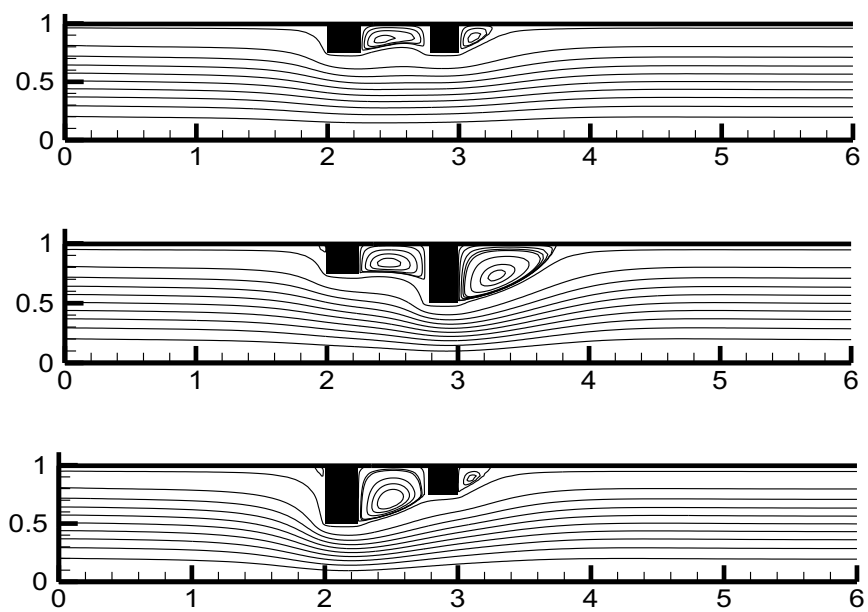

Fig. 5. Streamlines for the three combinations. $R e=100$, $\operatorname{Pr}=10, n=1$.

and the intensity of the recirculation zones between the blocks is slightly affected by the power law index. However, the recirculation zone behind the second block is very sensitive to the variation of the power law index and particularly as regards its size which increases noticeably with the decrease of the power law index.

\section{Effect of the obstacle height on the Nusselt number}

Concerning the thermal aspect, the heat transfer rate is characterized by the value of the local Nusselt number as a function of the obstacle peripheral distance. Figures 7-9 display the local Nusselt number along the two obstacles surfaces for different height for the $3 n$-values, correspondingly.

We observe that the Nusselt number is maximal in the left corner of the first obstacle (B1), for the three combinations. We note also for the case of combination
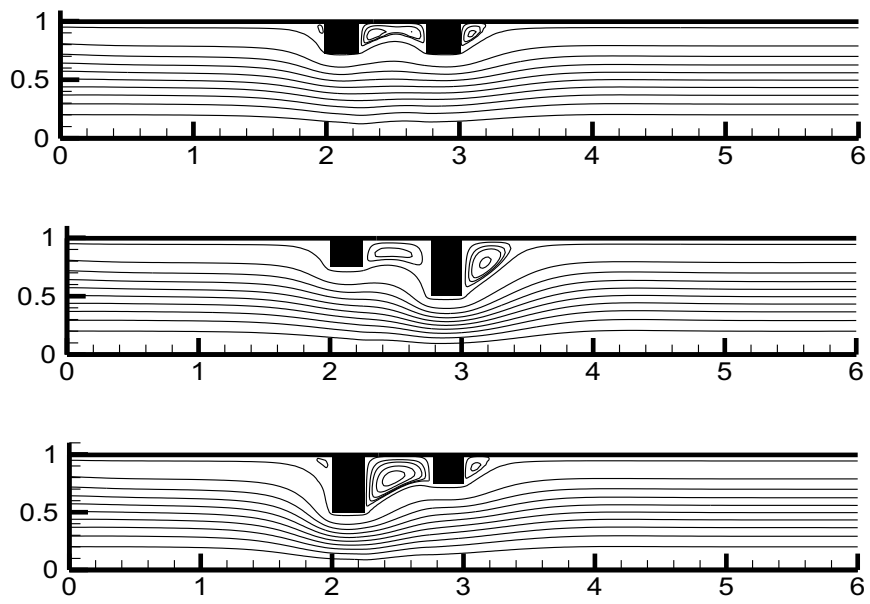

Fig. 6. Streamlines for the three combinations. $R e=100$, $\operatorname{Pr}=10, n=1.5$.

(b) that the Nusselt number is more important on the face (B1C1) for the first obstacle because of the increase of the velocity in this area. However, it decreases on the right face of the first obstacle (C1D1) where the recirculation zone has high intensity.

On the left face of the second obstacle (C2D2), the Nusselt number is similar for the two combinations (a) and (b) since the recirculation zone has the same intensity for both cases. Otherwise, when the size of the second obstacle increases, heat transfer on the face $(\mathrm{B} 2 \mathrm{C} 2)$ is improved as the velocity increases.

From a phenomenological point of view, the Nusselt number variations are not affected by the flow index. However, the values are significantly important for a shearthinning fluid $(n=0.5)$ compared to a Newtonian $(n=1)$ and shear-thickening $(n=1.5)$ fluid.

\section{Effect of obstacle distance}

The streamlines in Figure 10 reveal that there is recirculation zones between the two obstacles and downstream the second one. It can be seen that the size of the recirculation zone located between the two obstacles increases with the increase of the distance $\mathrm{S}$ between these two obstacles without breaking, even if $S=3 \mathrm{~W}$.

Indeed, the extent of this recirculation zone depends on the space offered; more the distance $\mathrm{S}$ is great, more the recirculation zone extends in the longitudinal direction.

For the Newtonian fluid, presented in Figure 11, we notice a recirculation zone between the two obstacles and behind the second one. When the separation distance increases, the size of the recirculation zone increases downstream of the first obstacle and occupy more and more the space between the two obstacles. However, from a distance corresponding to $S=3 \mathrm{~W}$, this recirculation zone occupies a portion of this space (near the face C1D1). In this region, an important heat removal is provided because of the important mixing. In contrast, the size of the recirculation zone downstream the second obstacle stays the same. 

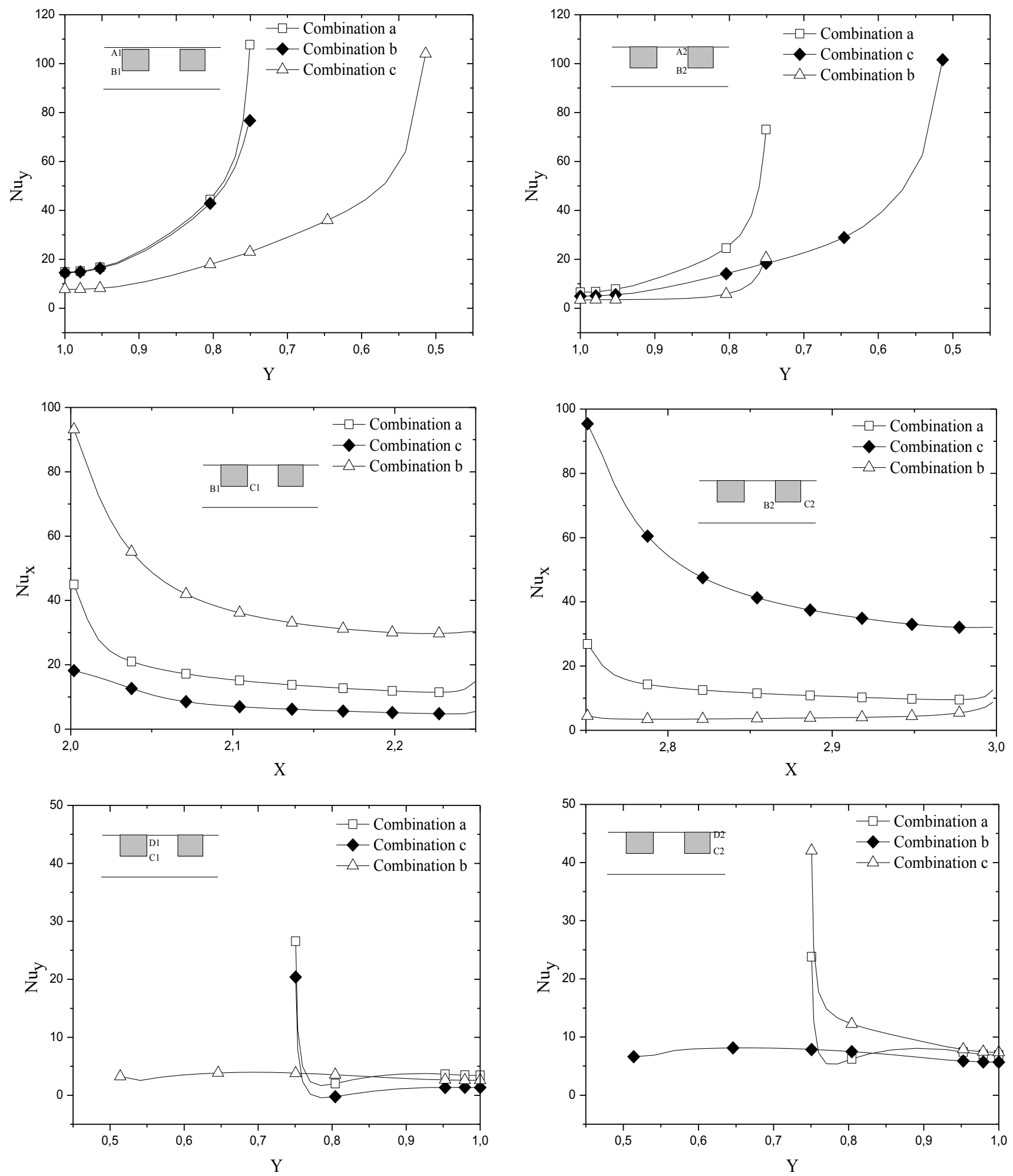

Fig. 7. Nusselt number along the faces of the two obstacles for the three combinations. $\operatorname{Re}=100, \operatorname{Pr}=10, n=0.5$.

Figure 12 shows the flow pattern for $n=1.5$. We get for this type of fluid the appearance of a third recirculation zone with low intensity upstream of the first obstacle in addition to other two: between both obstacles and downstream of the second obstacle. Furthermore, for a distance $S<3 W$, a single recirculation zone occupies the space between the two obstacles, with a tendency to be divided into two recirculation zones, clearly visible when $S=2 W$ (case (b)) and is confirmed when $S=3 W$ (case (c)). Indeed, for the latter case, the central cell ended up 

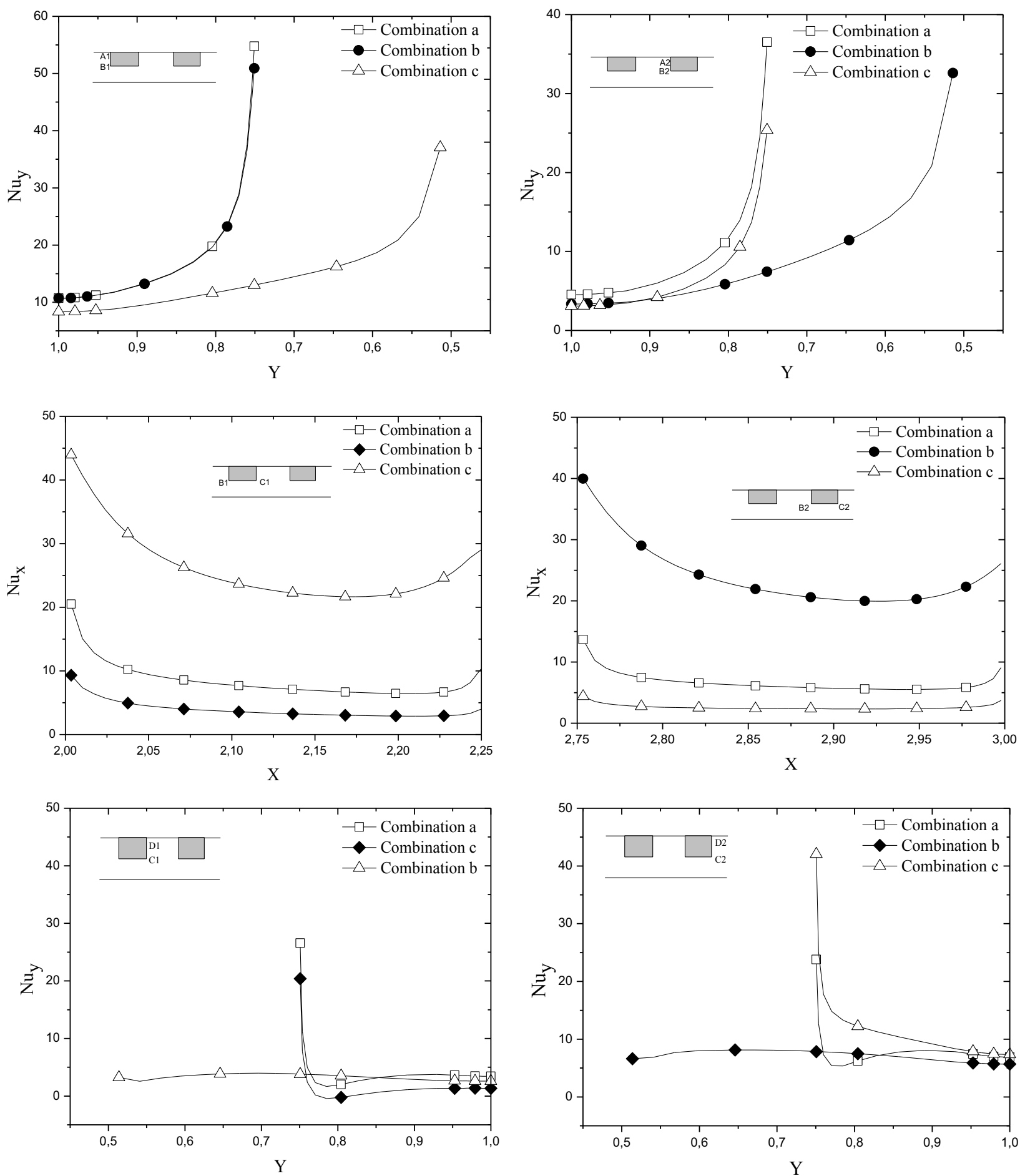

Fig. 8. Nusselt number along the faces of the two obstacles for the three combinations. $R e=100, \operatorname{Pr}=10, n=1$.

giving birth to two cells, located in the immediate vicinity of the two blocks, which rotate in an anticlockwise direction.

In Figure 13 is presented the variation of the Nusselt number according to the obstacle distance. We note that the influence of the separation distance is felt only on the face C1D1 of the first obstacle as well as on the faces A2B2 and $\mathrm{B} 2 \mathrm{C} 2$ of the second obstacle where the Nusselt number increases with the increase of the distance between the two obstacles.

At first glance, the values of the local Nusselt number calculated at the first obstacle are much higher compared to the second obstacle. In addition, the influence of the separation distance is felt only on the face $\mathrm{C} 1 \mathrm{D} 1$ of the first obstacle and on faces $\mathrm{A} 2 \mathrm{~B} 2$ and $\mathrm{B} 2 \mathrm{C} 2$ of the second obstacle, where the Nusselt number increases with the 

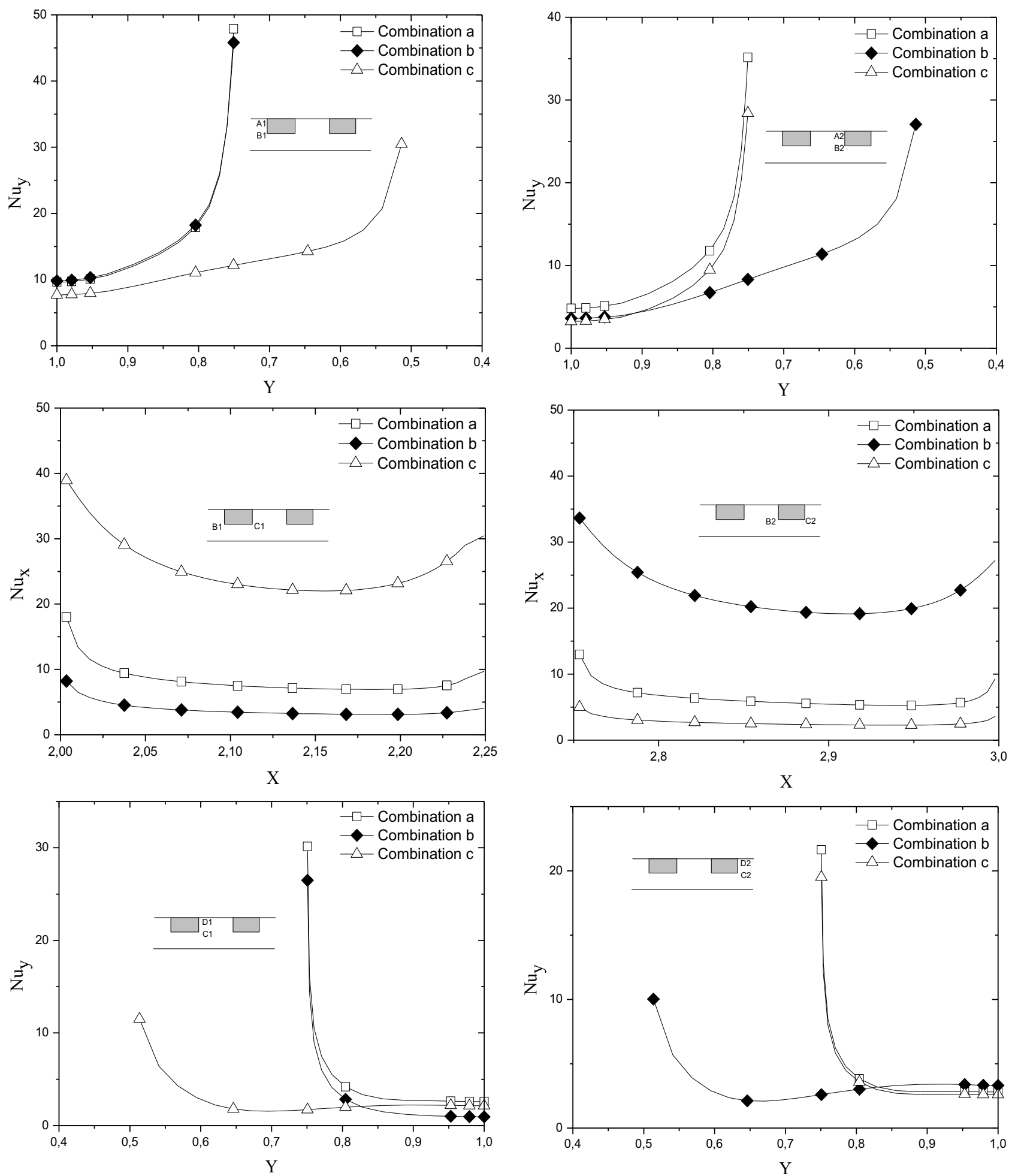

Fig. 9. Nusselt number along the faces of the two obstacles for the three combinations. $\operatorname{Re}=100, \operatorname{Pr}=10, n=1.5$.

separation distance $\mathrm{S}$. The increase can be explained by the increase of the intensity of the recirculation zone between the two obstacles.

The effect of the distance between the two obstacles on heat transfer for the case of a flow index $n=1$ is shown in Figure 14. As in the case of shear-thinning fluid, the heat transfer rate at the first obstacle is more important than at the second one. In addition, it is found that increasing the separation distance results in the increase of heat removal rates on faces $\mathrm{C} 1 \mathrm{D} 1, \mathrm{~A} 2 \mathrm{~B} 2$ and $\mathrm{B} 2 \mathrm{C} 2$, while the other faces remain insensitive to changes of the separation distance S. 

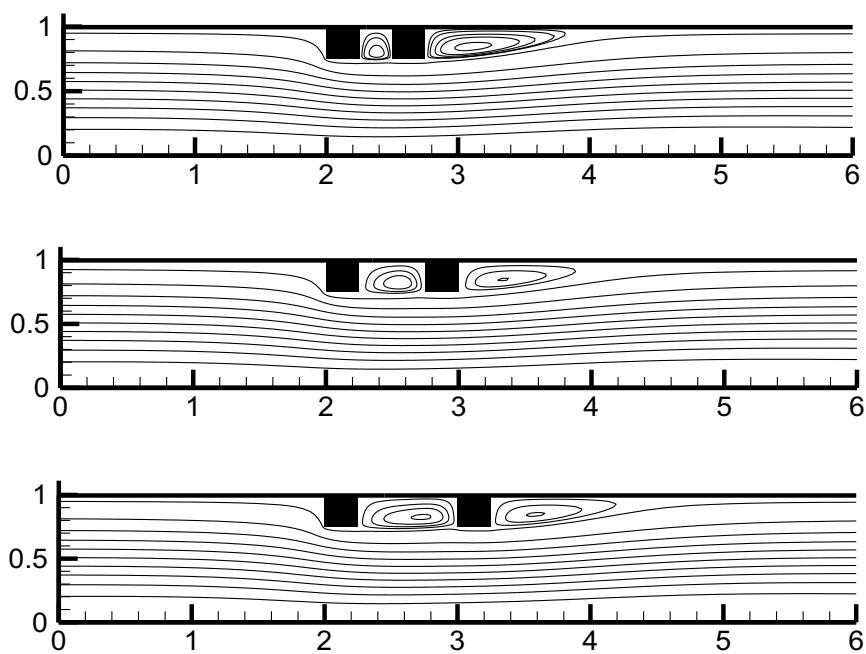

Fig. 10. Streamlines for the three separation distances. $R e=$ 100, $\operatorname{Pr}=10, n=0.5$.


Fig. 11. Streamlines for the three separation distances. $R e=100, \operatorname{Pr}=10, n=1$.

In addition, we note that the rate of heat removal has a maximum on the left corner of the first obstacle (B1) and that by increasing the value of the space between the two obstacles, the heat transfer is improved especially at the second obstacle.

It is clear from Figure 15 that the values of the local Nusselt number are generally the lowest for this type of fluid $(n=1.5)$ compared to the two previous cases $(n=0.5$ and $n=1)$. Similarly, as in the two previous cases, the influence of the separation distance is shown at the faces C1D1 of the first obstacle and A2B2 of the second one more than at the face $\mathrm{B} 1 \mathrm{C} 1$ of the first obstacle and $\mathrm{B} 2 \mathrm{C} 2$ of the second obstacle.

\section{Conclusion}

The present investigation concerned a laminar forced convection heat transfer of a non-Newtonian fluid flow within a parallel plate channel in which, two heat
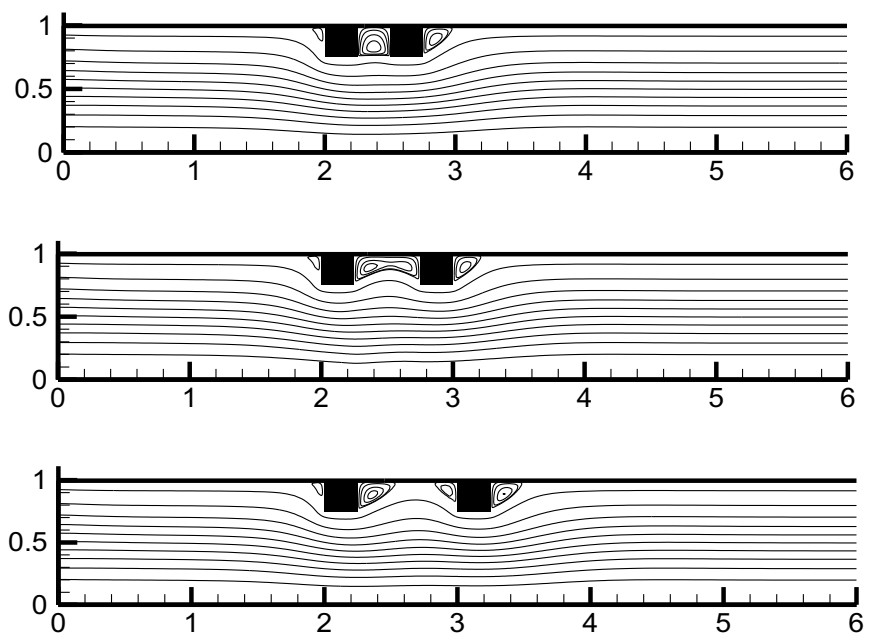

Fig. 12. Streamlines for the three separation distances $R e=100, \operatorname{Pr}=10, n=1.5$.
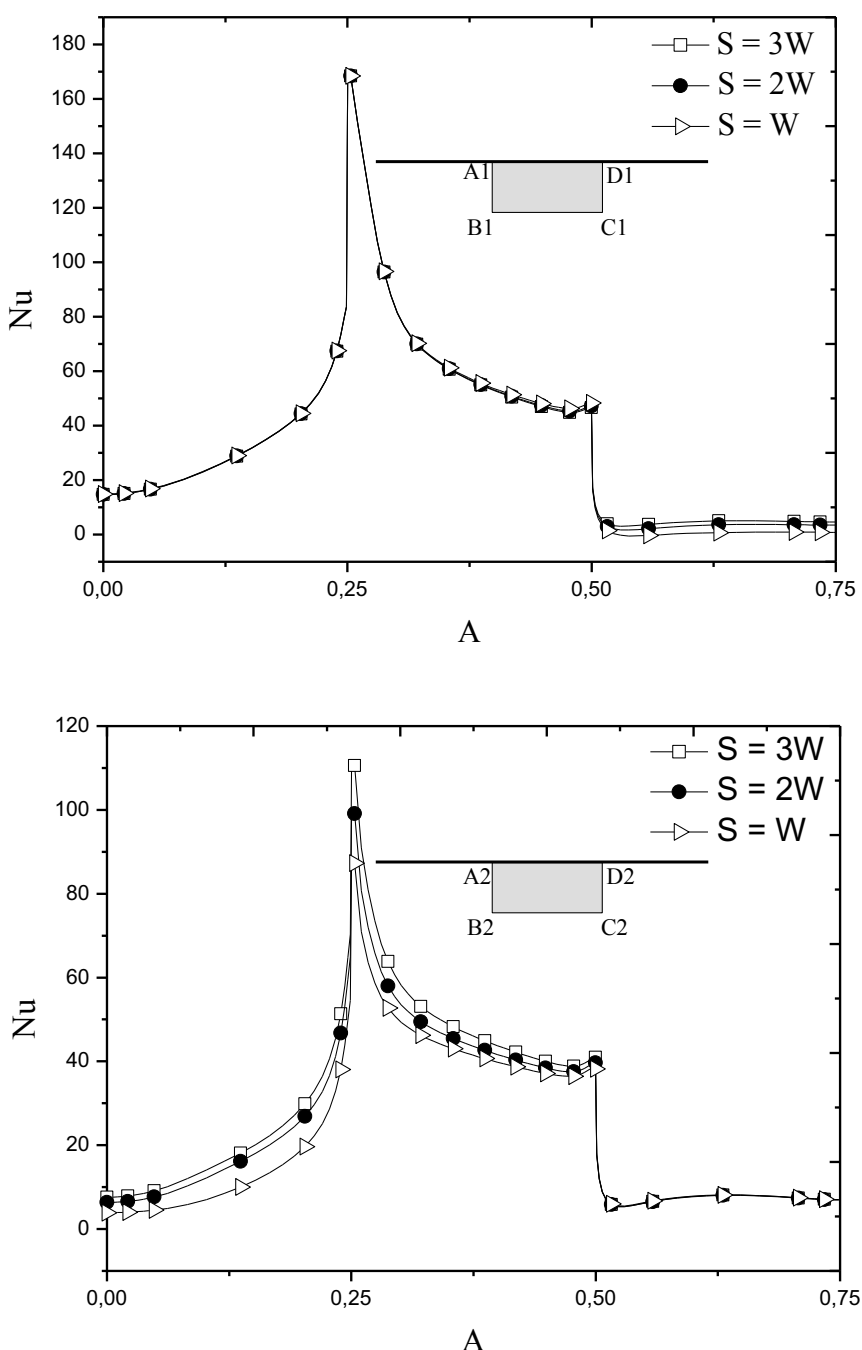

Fig. 13. Nusselt number evolution for the three separation distances. $R e=100, \operatorname{Pr}=10, n=0.5$. 

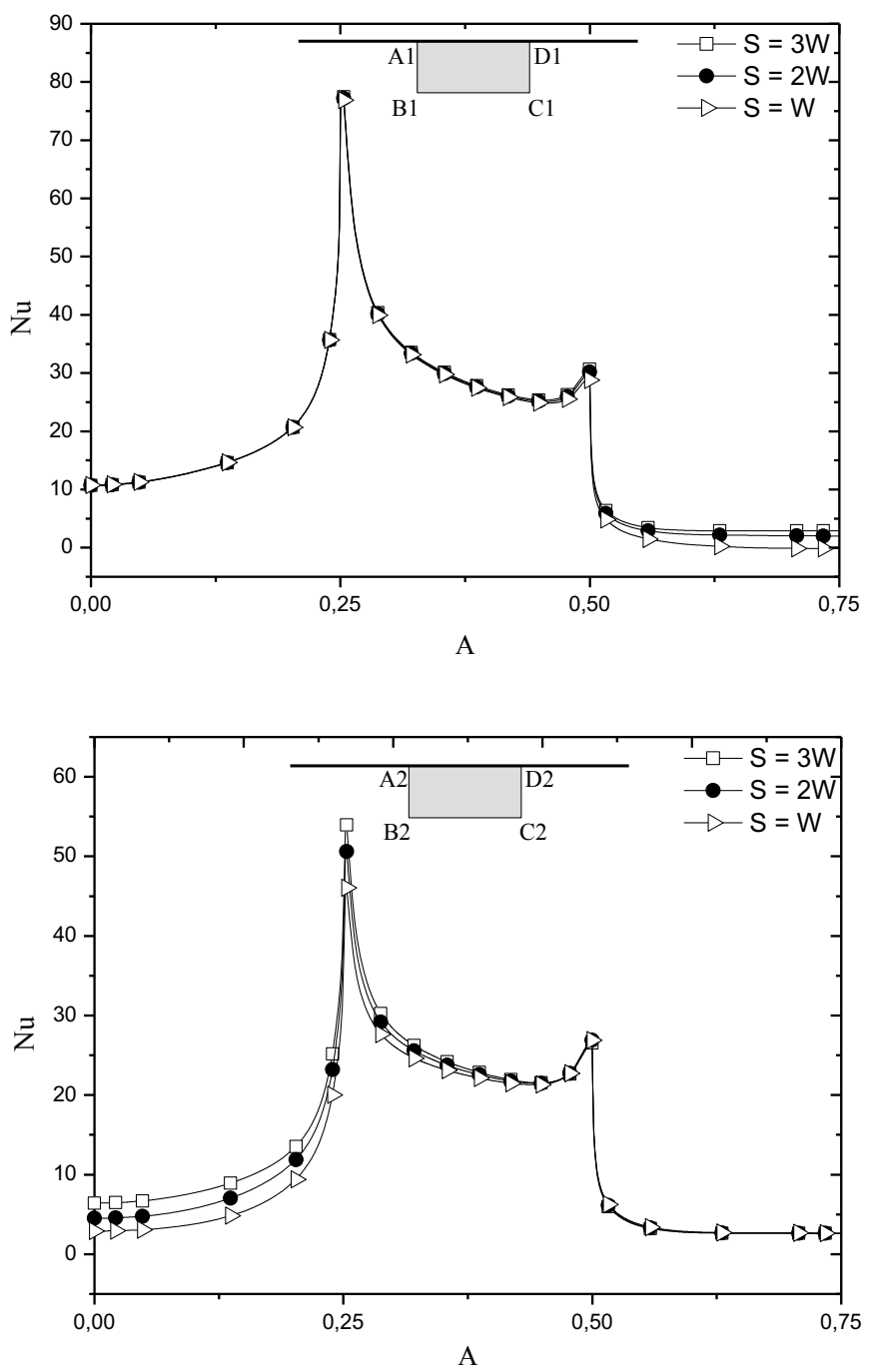

Fig. 14. Nusselt number evolution for the three separation distances. $R e=100, \operatorname{Pr}=10, n=1$.

generating obstacles are attached to the upper wall. We have selected three combinations for different height and separation distance of obstacles in order to analyze the fluid flow and heat transfer properties.

We found that varying the height of one of these two obstacles affect greatly the flow and heat transfer characteristic. Indeed, we observed two recirculation zones situated between the two obstacles and downstream the second obstacle. These recirculation zones have an important intensity downstream the higher obstacle. In addition, the heat transfer increased with the size of obstacle because of the increase of the velocity.

On the other hand, different separation distances were examined upon the fluid and heat transfer. The results showed that increasing the separation distance leads to increase the Nusselt number on the right side of the first obstacle and the left side of the second one.
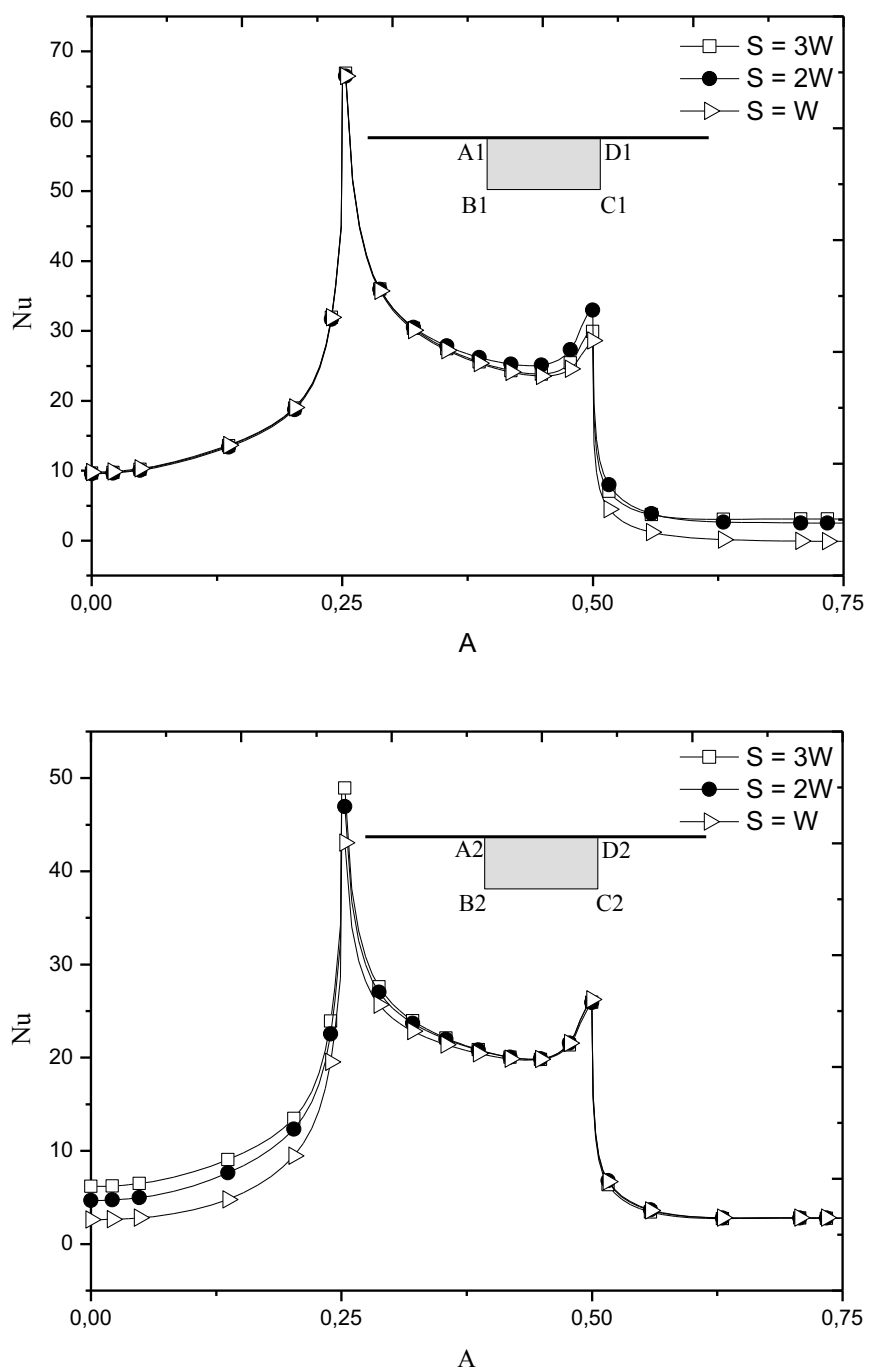

Fig. 15. Nusselt number evolution for the three separation distances. $R e=100, \operatorname{Pr}=10, n=1.5$.

Furthermore, we observed the formation of recirculation zones between the two obstacles and downstream of the second one.

We also found that for a separation distance $S<3 \mathrm{~W}$, the recirculation zone between the two obstacles occupies the entire space between them, whereas, for $S=3 \mathrm{~W}$, this area occupies a part (adjacent to the first obstacle) of the space between the two obstacles.

\section{Nomenclature}

$C$ Dimensionless block height

$H$ Dimensionless channel height

$k$ Thermal conductivity, $\mathrm{W} \cdot \mathrm{m}^{-2} \cdot \mathrm{K}^{-1}$

$K$ Thermal conductivity ratio

$L \quad$ Dimensionless channel length

$P^{*} \quad$ Dimensionless pressure 
$\operatorname{Pr} \quad$ Prandtl number, $\mu \cdot \mathrm{C}_{\mathrm{p}} \cdot \mathrm{k}_{\mathrm{f}}^{-1}$

$\begin{array}{ll}Q & \text { Heat flux, } \mathrm{W} \cdot \mathrm{m}^{-2} \\ R e & \text { Reynolds number, } \rho \cdot \mathrm{U}_{0} \cdot \mathrm{D} \cdot \mu^{-1}\end{array}$

$S$ Dimensionless spacing between the blocks

$u \quad$ Axial velocity, $\mathrm{m} \cdot \mathrm{s}^{-1}$

$v \quad$ Transverse velocity, $\mathrm{m} \cdot \mathrm{s}^{-1}$

$U$ Dimensionless axial velocity

$V \quad$ Dimensionless transverse velocity

$U_{0} \quad$ Mean velocity, $\mathrm{m} \cdot \mathrm{s}^{-1}$

$W$ Dimensionless block width

$x \quad$ Axial coordinate, $\mathrm{m}$

$y \quad$ Transverse coordinate, $\mathrm{m}$

$X$ Dimensionless axial coordinate

$Y$ Dimensionless transverse coordinate

\section{Greek letters}

$\lambda$ Constant used in the energy equation

$\mu$ Dynamic viscosity, $\mathrm{Pa} \cdot \mathrm{s}$

$\rho$ Mass density, $\mathrm{kg} \cdot \mathrm{m}^{-3}$

\section{Upper/lower case}

e Inlet

f Fluid

s Solid

* Dimensionless viscosity

\section{References}

[1] T.J. Young, K. Vafai, Convective cooling of a heated obstacle in a channel, Int. J. Heat Mass Transf. 41 (1998) 3131-3148
[2] T.J. Young, K. Vafai, Convective flow and heat transfer in a channel containing multiple heated obstacles, Int. J. Heat Mass Transf. 41 (1998) 3279-3298

[3] M.H. Yang, R.H. Yeh, J.J. Hwang, Forced convective cooling of a fin in a channel, Energy Convers. Manag. 51 (2010) 1277-1286

[4] A. Hamouche, R. Bssaih, Mixed convection air cooling of protruding heat sources mounted in a horizontal channel, Int. Commun. Heat Mass Transf. 36 (2009) 841-849

[5] S.Y. Kim, B. Hakang, J.M. Hyun, Forced convection heat transfer from two heated blocks in pulsating channel flow, Int. J. Heat Mass Transf. 41 (1998) 625-634

[6] A. Korichi, L. Oufer, Numerical heat transfer in a rectangular channel with mounted obstacles on upper and lower walls, Int. J. Therm. Sci. 44 (2005) 644-655

[7] M. Mohammadi Pirouz, M. Farhadi, K. Sedighi, H. Nemati, E. Fettahi, Lattice Boltzmann simulation of conjugate heat transfer in a rectangular channel with wall-mounted obstacles, Sci. Iran. B 18 (2011) 213-221

[8] H.W. Wu, SW. Perng, Effect of an oblique plate on the heat transfer enhancement of mixed convection over heated blocks in a in a horizontal channel, Int. J. Heat Mass Transf. 42 (1999) 1217-1235

[9] H.F. Oztop, Y. Varol, D.E. Alnak, Control of heat transfer and fluid flow using a triangular bar in heated blocks located in a channel, Int. Commun. Heat Mass Transf. 36 (2009) 878-885

[10] S.W. Perng, H.W. Wu, Numerical investigation of mixed convective heat transfer for unsteady turbulent flow over heated blocks in a horizontal channel, Int. J. Therm. Sci. 47 (2008) 620-632

[11] R.M. Cotta, M.N. Ozisik, Laminar forced convection of power-law non-Newtonian fluids inside ducts, Warme-und Stoffubertragung 20 (1986) 211-218

[12] R. Nebbali, K. Bouhadef, Non-Newtonian fluid flow in plane channels: Heat transfer enhancement using porous blocks, Int. J. Therm. Sci. 50 (2011) 1984-1995

[13] S.V. Patankar, Numerical heat transfer and fluid flow, Hemisphere Corp, Washington, 1980

Cite this article as: A. Boudiaf, F. Danane, Y.K. Benkahla, N. Labsi, A. Boutra, Heat transfer convection of a power law fluid flow within a parallel plate channel provided with two generating obstacles, Mechanics \& Industry 19, 210 (2018) 